\title{
The motivation to reproject gated blood pool SPECT data as planar data
}

\author{
Kenneth J. Nichols, PhD, ${ }^{a}$ and Denny D. Watson, $\mathrm{PhD}^{\mathrm{b}}$
}

See related article, pp. 358-366

In the current issue of the Journal, Wells et al, describe the results of deriving left ventricular (LV) ejection fraction $(\mathrm{EF})$ values from planar radionuclide ventriculography (RNV) images that are not conventionally obtained via best septal view planar imaging, but rather by reprojecting gated blood pool SPECT data as planar RNV images. ${ }^{1}$ This is a topic of relevance for laboratories having SPECT-only cameras which are requested to perform gated blood pool scans.

The most common reason for requesting RNV studies is to monitor possible cardiotoxicity caused by chemotherapeutic agents, such as the use of anthracycline-based chemotherapy to treat breast carcinoma in adults $^{2}$ and to treat Hodgkin disease (HD) in children. ${ }^{3}$ Serial RNV studies of EF during therapy provide a sufficient means of avoiding drug-induced congestive heart failure. ${ }^{4}$ Some published guidelines for evaluating anthracycline cardiotoxicity in children advocate using both echocardiography and planar RNV studies. ${ }^{5}$ However, as treatment protocols can extend over several weeks or months, serial evaluations of EF are required, so that in general serial measurement of EF for children is performed by echocardiography, ${ }^{5}$ but for adults is performed by planar RNV as it has been considered the best non-invasive EF method. ${ }^{6}$ Subjects can be followed for 10 years or more because features of abnormal cardiac function will sometimes appear only many years later after completion of treatment. ${ }^{7,8}$ Reproducibility is therefore critical for serial studies of patients undergoing chemotherapy. Changes in EF as small as $10 \%$ may be interpreted as clinically significant. These changes are

From the Division of Nuclear Medicine \& Molecular Imaging, ${ }^{\text {a }}$ North Shore-Long Island Jewish Health System, New Hyde Park, NY and University of Virginia, ${ }^{\text {b }}$ Charlottesville, VA

Reprint requests: Kenneth J. Nichols, PhD, Division of Nuclear Medicine \& Molecular Imaging, North Shore-Long Island Jewish Health System, 270-05, 76th Avenue, New Hyde Park, NY 11040; knichols@lij.edu.

J Nucl Cardiol 2013;20:329-30.

$1071-3581 / \$ 34.00$

Copyright $@ 2013$ American Society of Nuclear Cardiology.

doi:10.1007/s12350-013-9698-y not accompanied by corroborative symptoms and decisions to initiate or withhold potentially life-saving therapy may be based solely on the EF values.

So, what is one to do now, in 2013 , in a laboratory without a planar gamma camera, but with a solid state detector that can only perform SPECT, not planar, imaging? How does one relate an EF value obtained by this newer technology to serial EF values obtained by the older planar RNV studies acquired with Anger cameras?

Planar EFs have long been the desired means of evaluating EFs because of the simplicity of the imaging conditions. Planar RNV imaging also provides diastolic filling parameters, which progressively deteriorate with anthracycline therapy, along with phase histograms and fairly robust visual images that can be used for quality control and to corroborate significant changes in the value of EF. In terms of acquiring images, it is relatively undemanding to identify the angle for the best planar septal LAO view. In terms of processing acquired data, automated algorithms are often successful at generating LV and background regions that are satisfactory, but when they require manual correction, the process of manually drawing a ROI to isolate LV activity is fairly straightforward. So, it is the simplicity of the imaging setup in combination with the simplicity of data analysis that facilitates reproducible EF values. It is considered feasible to obtain planar EF values with a reproducibility of $4-5 \%$. $^{9,10}$

No test is perfect, and the shortcomings of planar RNV imaging have long been recognized. Because red blood cells in the lungs in front of and behind the LV contribute to cross talk counts, along with scattered radiation from other non-ventricular structures, counts in the ROI intended to isolate the LV may be contaminated. Subtracting average background counts as estimated using neighboring regions helps to correct for tissue cross talk, but the major impediment to planar RNV EF absolute accuracy is variability from one patient to another in the degree of contamination by atrial counts overlapping LV counts. Patients have a range of relatively large to relatively small $\mathrm{LV}$ atria, which are variably located in relation to the LV. Frequently, it is not possible to distinguish the LA visually with any degree of confidence. This is the reason that has most frequently been cited as to why $2 \mathrm{D}$ planar RNV 
EFs are not identical to 3D SPECT RNV EFs, such that these are not interchangeable. ${ }^{11}$ SPECT RNV EFs nevertheless appear to be accurate. Comparisons between SPECT RNV and cardiac MRI EFs have found statistically similar values, with Pearson correlation coefficients ranging from $r=0.89-0.95 .{ }^{12,13}$ In addition, SPECT RNV EF calculations that are based on count thresholds are as reproducible as planar RNV EFs. ${ }^{14}$

So, for a laboratory having only solid state SPECTcapable detectors which is requested to assess EF for a patient who has previously had a succession of planar RNV studies, there appears to be no reliable way of obtaining comparable EFs. Wells et al, offer a solution in that one can produce two EFs: a SPECT EF value comparable to what would have been obtained by MRI and another planar EF value derived by reprojecting SPECT data that should be comparable to what would have been obtained with a planar gamma camera.

Referrals of patients having chemotherapy will likely grow as the number of patients increases and considering that many of the newer classes of chemotherapy drugs also have a spectrum of cardiotoxic effects. A valuable aspect of Wells' work is that it may open a pathway for Nuclear Cardiology practitioners to bridge the gap between their serial EF values previously obtained with planar cameras and the possibility of performing all future serial RNV studies as SPECT RNV with solid state cameras.

The work of Wells et al also leads us to reflect that while the reproducibility of these methods (with standard errors of $4-5 \%$ ) in a controlled research setting appears to be just adequate for monitoring chemotherapy, the consequences of spurious error in this setting are potentially quite serious. That there are no corroborating clinical manifestations imposes demands on a method being considered as a replacement to an established technique that is very robust in average clinical laboratories. As we integrate newer technologies, this may be a good time to further develop new study designs and to critically evaluate the accuracy, reproducibility, and robustness of the various methods of following patients having chemotherapy and to reevaluate the appropriateness of all EF methods.

\section{References}

1. Wells RG, Marvin B, Kovalski G, D Ruddy TD. Planar radionuclide angiography with a dedicated cardiac SPECT camera. J Nucl Cardiol 2013. doi:10.1007/s12350-013-9674-6
2. Feola M, Garrone O, Occelli M, Francini A, Biggi A, Visconti G, et al. Cardiotoxicity after anthracycline chemotherapy in breast carcinoma: Effects on left ventricular ejection fraction, troponin I and brain natriuretic peptide. Int J Cardiol 2011;148:194-8.

3. Tantawy AAG, Elmasry OA, Shaaban M, Toaima DA, El Shahat AM. Radionuclide ventriculography detects early anthracycline cardiotoxity in children with Hodgkin lymphoma. J Pediatr Hematol Oncol 2011;33:e132-7.

4. Mitani I, Jain D, Joska TM, Burtness B, Zaret BL. Doxorubicin cardiotoxicity: Prevention of congestive heart failure with serial cardiac function monitoring with equilibrium radionuclide angiocardiography in the current era. J Nucl Cardiol 2003;10:132-9.

5. Steinherz LJ, Graham T, Hurwitz R, et al. Guidelines for cardiac monitoring of children during and after anthracycline therapy: report of the cardiology committee of the Children's Cancer Study Group. Pediatrics 1992;89:942-9.

6. Ganz WI, Sridhar KS, Ganz SS, Gonzalez R, Chakko S, Serafini A. Review of tests for monitoring doxorubicin-induced cardiomyopathy. Oncology 1996;53:461-70.

7. Lipshultz SE, Colan SD, Gelber RD, Perez-Atayde AR, Sallan SE, Sanders SP. Late cardiac effects of doxorubicin therapy for acute lymphoblastic leukemia in childhood. NEngl J Med 1991;324:808-15.

8. Vandecruys E, Mondelaers V, De Wolf D, Benoit Y, Suys B. Late cardiotoxicity after low dose of anthracycline therapy for acute lymphoblastic leukemia in childhood. J Cancer Surviv 2012;6:95101.

9. Hecht HS, Josephson MA, Hopkins JM, Singh BN. Reproducibility of equilibrium radionuclide ventriculography in patients with coronary artery disease: Response of left ventricular ejection fraction and regional wall motion to supine bicycle exercise. Am Heart J 1982;104:567-74.

10. Mahmarian JJ, Moye L, Verani MS, Eaton T, Francis M, Pratt CM. Criteria for the accurate interpretation of changes in left ventricular ejection fraction and cardiac volumes as assessed by rest and exercise gated radionuclide angiography. J Am Coll Cardiol 1991;18:112-9.

11. Bartlett ML, Srinivasan G, Barker WC, Kitsiou AN, Dilsizian V, Bacharach SL. Left ventricular ejection fraction: Comparison of results from planar and SPECT gated blood-pool studies. J Nucl Med 1996;37:1795-9.

12. Nichols KJ, Van Tosh A, Wang Y, Palestro CJ, Reichek N. Validation of gated blood-pool SPECT regional left ventricular function measurements. J Nucl Med 2009;50:53-60.

13. Xie B-Q, Tian Y-Q, Zhang J, Zhao S-H, Yang M-F, Guo F, et al. Evaluation of left and right ventricular ejection fraction and volumes from gated blood-pool SPECT in patients with dilated cardiomyopathy: Comparison with cardiac MRI. J Nucl Med 2012;53:584-91.

14. Daou D, Coaguila C, Benada A. Comparison of interstudy reproducibility of equilibrium electrocardiography-gated SPECT radionuclide angiography versus planar radionuclide angiography for the quantification of global left ventricular function. J Nucl Cardiol 2006;13:233-43. 